Evaluar, 8 (2008), 46 - 60

\title{
Adaptación del Inventario Alemán de Ansiedad frente a los Exámenes: GTAI-A
}

\author{
Heredia Daniel, ${ }^{1 *}$ Piemontesi Sebastián*, Furlan Luís* \& Volker Hodapp** \\ * Laboratorio de Evaluación Psicológica y Educativa, Universidad Nacional de Córdoba \\ **Universidad Johann Wolfgang Goethe de Frankfurt, Alemania.
}

Resumen. El objetivo de esta investigación fue adaptar el inventario alemán de ansiedad frente a los exámenes (TAI-G) para su empleo en estudiantes universitarios argentinos. El TAI-G (Hodapp, 1991) es un autoinforme de 30 ítems, agrupados en cuatro escalas: Preocupación, Emocionalidad, Interferencia y Falta de Confianza. Se utilizó el método de traducción inversa, y una primera versión en español fue administrada a 347 estudiantes de diferentes carreras de la Universidad Nacional de Córdoba, Argentina. El Análisis Factorial Exploratorio permitió obtener una solución que conservó la estructura original de cuatro dimensiones, pero con índices moderados de consistencia interna en algunas escalas. Por esta razón, se reformularon algunos ítems y el instrumento fue nuevamente administrado a una muestra de 212 estudiantes. En este nuevo estudio, los niveles de consistencia interna fueron adecuados dando como resultado un inventario de 28 ítems denominado GTAIA. Finalmente, se obtuvieron evidencias adicionales de validez en relación a género y rendimiento académico.

Palabras clave. Ansiedad frente a los exámenes, Análisis Factorial Exploratorio, Adaptación de Tests.

\begin{abstract}
The German Test Anxiety Inventory (TAI-G) was adapted to be used on Argentinean university students. The TAI-G (Hodapp, 1991) is a self-report with 30 items grouped into fourth scales: Worry, Emotionality, Interference and Lack of Confidence. The items were back translated from English to Spanish and the first Spanish version was administered to 347 students from different careers of the National University of Cordoba, Argentina. The Exploratory Factor Analysis supported the original fourth-factor structure, but with moderated internal consistency levels. For this reason, the weak items were reformulated and administrated to 212 students. Internal consistency levels improved resulting in a 28 items inventory called GTAI-A. Also was analyzed external validity in relation to gender and academic performance.
\end{abstract}

Keywords. Test anxiety, Exploratory Factor Analysis, Test Adaptation.

\footnotetext{
${ }^{1}$ Por favor dirigir la correspondencia relacionada con este artículo a:

Daniel Heredia

Laboratorio de Evaluación Psicológica y Educativa. Facultad de Psicología Universidad Nacional de Córdoba.

Enrique Barros y Enfermera Gordillo. Ciudad Universitaria. (5000) Córdoba. Teléfono: 54-351-4822227.

Correo electrónico: dheredia@psyche.unc.edu.ar
} 


\section{Introducción}

La ansiedad frente a los exámenes puede definirse como un rasgo especificosituacional, caracterizado por la predisposición a reaccionar con elevada ansiedad en contextos que se relacionan con el rendimiento (Hodapp, Glazman, \& Laux, 1995). Para Gutiérrez Calvo y Avero, (1995) constituye una tendencia relativamente estable de algunos individuos a responder con elevados niveles de ansiedad ante situaciones en las que sus aptitudes están siendo evaluadas, cuyo aspecto central es la preocupación por el posible mal desempeño en la tarea y sus consecuencias aversivas para la autoestima, el estatus o la pérdida de algún beneficio esperado.

Si bien los primeros estudios sobre la ansiedad frente a los exámenes se iniciaron hace más de 90 años (Folin, Demis, \& Smillie, 1914), las investigaciones sistemáticas se remontan recién a 1952 cuando Sarason y Mandler exploraron sus relaciones con el rendimiento y elaboraron un instrumento para adultos llamado Test Anxiety Questionnaire (TAQ). En un principio, la ansiedad frente a los exámenes se consideró un constructo unidimensional, es decir, una respuesta global y unitaria que incluía manifestaciones cognitivas, somáticas y conductuales. En las décadas de los 60 y 70 hubo dos avances fundamentales en su conceptualización. Por un lado, la distinción entre la ansiedad como estado y como rasgo estable de la personalidad (Cattell \& Scheier, 1961; Spielberger, 1966); y por el otro, la distinción entre dos dimensiones básicas de la experiencia ansiógena, una de carácter cognitivo llamada Preocupación (Worry) y otra de tipo afectivo - fisiológico denominada Emocionalidad (Emotionality). Este enfoque se denominó Modelo Dual. (Liebert \& Morris, 1967).

Tres inventarios ampliamente utilizados se elaboraron siguiendo los postulados de este Modelo: el Worry Emotionality Questionnaire (WEQ; Liebert \& Morris, 1967); el Test Anxiety Scale (TAS; Sarason, 1978) y el Test Anxiety Inventory (TAI, Spielberger, 1980). Estos cuestionarios poseen diferentes cantidades de ítems y formatos de respuesta aunque todos emplean indicadores semejantes y permiten obtener puntuaciones parciales para las escalas preocupación y emocionalidad y un puntaje total. Otro aspecto común es que conceptualizan y permiten medir la ansiedad ante los exámenes como un rasgo situacional específico, es decir, como una tendencia relativamente estable de los individuos a responder con ansiedad ante situaciones que posean carácter evaluativo y no necesariamente ante otras o indicando la presencia de un rasgo de ansiedad general. No obstante, se han formulado 
reiteradas críticas a este modelo, especialmente en lo relativo a la elevada superposición entre las dimensiones (Benson, 1993; Benson \& Tippets, 1990; Everson, Milsap, \& Rodríguez, 1991; Hocevar \& El-Zahhar, 1985; Ware, Galassi, Michie, \& Dew, 1990; Zeidner \& Nevo, 1992; Zimmer, Hocevar, Bachelor, \& Meinke, 1992).

A partir de los 80 se crearon nuevas escalas para incorporar los aportes derivados de reformulaciones teóricas del modelo dual. Asimismo, se incrementó el cuidado de los aspectos técnicos en los procesos de construcción, adaptación y validación, especialmente con el empleo del Análisis Factorial Confirmatorio. Diversos autores aportaron evidencias a favor del carácter multidimensional de la ansiedad frente a los exámenes y, en este sentido, la dimensión clásica "Preocupación" fue reconocida como un constructo más heterogéneo y diferenciado (Heckhausen, 1982; Salamé, 1984; Schwarzer \& Quast, 1985; Stephan, Fischer, \& Stein, 1983, Rost \& Schermer, 1989; Wine, 1982).

En este marco Sarason (1984) propuso una medida multidimensional de la ansiedad frente a los exámenes, el Reaction to Test (RTT) que diferenció en la dimensión cognitiva de la ansiedad, la preocupación y los pensamientos irrelevantes, y dentro de la emocionalidad, la tensión y los síntomas corporales. La distinción entre Preocupación y Pensamientos Irrelevantes se relaciona con el mecanismo de interferencia cognitiva que explicaría la disminución del rendimiento a través del proceso caracterizado como pensamientos intrusivos que desvían la atención de la tarea que se esta realizando (Wine, 1971).

El RTT presentó algunos problemas psicométricos, tales como la escasa consistencia interna de las subescalas y las bajas correlaciones entre las mismas. En un intento de revertir estas falencias, se elaboró una versión abreviada del RTT resolviendo solo parcialmente estos inconvenientes (Benson \& Bandalos, 1992). Por este motivo, se construyó un nuevo instrumento que conserva la estructura interna del RTT pero con mejores propiedades psicométricas. La Revised Test Anxiety Scale (RTA) combinó ítems del TAI y del RTT y demostró poseer una estructura interna clara y estable, apoyada en Análisis Confirmatorios realizados en muestras transculturales de estudiantes (Benson \& EI-Zahhar, 1994).

\section{German Test Anxiety Inventory: TAI-G.}

Hodapp (1991) desarrolló el TAI-G como una adaptación a la población alemana del Test Anxiety Inventory (Spielberger, 1980). Sin embargo, realizó modificaciones profundas en la estructura interna de la prueba original que era el aspecto con mayores problemas para 
la validación de constructo (Ware et al., 1990). Durante la adaptación del TAI al idioma alemán, Hodapp, Laux, y Spielberger (1982) observaron que algunos ítems del TAI eran muy complejos, puesto que incluían descripciones de la situación de examen así como de las respuestas ante la misma. Para la reformulación del TAI, en un primer momento se tomaron 78 ítems de varias escalas alemanas y norteamericanas de ansiedad frente a los exámenes, incluyendo los 20 originales del TAI. Todos los ítems fueron modificados para referirse solamente a sentimientos y cogniciones en situaciones de examen, eliminándose de esta manera las referencias temporales; aunque en varios cuestionarios ampliamente usados existen un número de ítems con referencias tales como "antes", "durante" o "después" de un examen, el problema con estas referencias es que la validez de un ítem puede ser diferente para las distintas etapas del ciclo de aprendizaje-examen. Los ítems modificados fueron administrados a 477 adolescentes de entre 13 y 16 años de edad. Un análisis factorial exploratorio diferenció los factores de Preocupación y Emocionalidad y la estructura se mantuvo estable cuando los datos fueron analizados separadamente entre géneros y diferentes colegios (Hodapp et al., 1995). A pesar de la adecuación psicométrica y apoyo empírico para la validación de esta adaptación (Hodapp, 1989; Hodapp \& Henneberger, 1982; Hodapp et al., 1982; Kerres, 1988; Liepmann, Margraff, Felfe, \& Hosemann, 1992; Schwarzer, 1984), algunas cuestiones adicionales estimularon la reconceptualización y expansión del alcance del TAI-G (Hodapp, 1991). Una de las razones fue que la adaptación del TAI-G incluía varios ítems relacionados con Confianza acerca del Rendimiento, dimensión que podría constituir un factor diferenciado. Por otra parte, algunas investigaciones habían sugerido que la Preocupación era un componente heterogéneo y diferenciado de la respuesta de ansiedad. De esta manera, se elaboraron 80 ítems que incluyeron los de la versión preliminar del TAI-G (Hodapp et al., 1982) y otros referidos a las dimensiones preocupación existencial, pensamiento autorreferencial, afrontamiento, anticipación de fracaso, confianza, pensamientos irrelevantes e interferencia. El conjunto de ítems se administro a 713 estudiantes realizándose luego análisis factorial, análisis de ítems y Rasch análisis (Hodapp, 1991). El análisis factorial exploratorio reveló una solución de cuatro factores que explicaron $45.4 \%$ de la varianza total: Emocionalidad $(\alpha=.88$, con ocho ítems referidos a las percepciones de la activación fisiológica), Preocupación $(\alpha=.87$, con diez ítems referidos a pensamientos sobre las consecuencias negativas de fracasar), Falta de Confianza ( $\alpha=.90 \mathrm{y}$ seis ítems acerca de la creencias negativas de la propia capacidad de rendir adecuadamente el 
examen); Interferencia $(\alpha=.85$, también con seis ítems relacionados con pensamientos que producen distracción y bloqueo cognitivo). Tanto la escala total $(\alpha=.93)$ como las subescalas demostraron consistencia interna satisfactoria. El análisis de Rasch proporcionó evidencia adicional de que las cuatro sub-escalas pertenecen a un mismo constructo. En conjunto, los resultados apoyan la conceptualización multidimensional de la ansiedad frente a los exámenes.

También se realizaron investigaciones en relación a la estabilidad temporal y las relaciones del TAI-G con otras variables, tales como género, rendimiento y estrategias de afrontamiento (Buchwald, 2002; Buchwald \& Schwarzer, 2004; Hodapp, 1995; Hodapp et al., 1995; Hodapp \& Benson, 1997; Keith, Hodapp, Schermelleh-Engel \& Moosbrugger; 2003; Musch \& Broder, 1999; Rost \& Schermer, 1997; Stöber, 2004).

Considerando estas evidencias sobre las bases teóricas y propiedades psicométricas del TAI-G y la necesidad de contar con un instrumento multidimensional para evaluar la ansiedad frente a los exámenes, decidimos realizar un primer estudio de traducción inversa de los ítems y administración a una muestra de 348 estudiantes de 17 carreras diferentes (mujeres 62\%, varones 35\%, media de edad=20.86). Un análisis factorial exploratorio (Ejes Principales, rotación Promax) nos permitió identificar una solución de 4 factores que explicaron un $42 \%$ de la varianza, con 25 ítems $(\alpha=.88)$ y cuatro escalas: Emocionalidad (8 ítems, $\alpha=.85$ ) Preocupación (7 ítems, $\alpha=.76$ ); Falta de Confianza (6 ítems, $\alpha=.86$ ); Interferencia (4 ítems, $\alpha=.67$ ). Se observó que algunos ítems presentaron correlaciones ítemfactor inferiores a $.35(6,10,19,23,30)$, así como un ítem bidimensional (15) (Heredia \& Piemontesi, 2008). Por otra parte, la consistencia interna de algunas escalas fue sólo moderada, especialmente Interferencia.

En consecuencia, el objetivo esencial de este trabajo es realizar nuevos estudios de adaptación del TAI-G que permitan mejorar las propiedades psicométricas de la versión adaptada a la población universitaria argentina.

\section{Método}

\section{1. Participantes y Procedimiento}

El instrumento fue administrado por algunos de los autores de este trabajo a una muestra accidental de 212 estudiantes (mujeres 83.5\%; varones 16.5\%; M=22,7 años) de la Facultad de Psicología de la Universidad Nacional de Córdoba, en horario regular de clases y 
con autorización de los profesores. La participación fue voluntaria y se enfatizó el carácter confidencial de los resultados.

\section{2. Instrumento}

La versión traducida al español hablado en Argentina del German Test Anxiety Inventory (TAI-G; Hodapp, 1991) es una medida de autoinforme que incluye 30 ítems con cuatro opciones de respuesta ("casi nunca" a "casi siempre"), distribuidos en cuatro escalas: Emocionalidad (8 ítems), Preocupación (10 ítems), Interferencia (6 ítems), y Falta de Confianza (6 ítems). Todos los ítems de las primeras tres escalas son codificados sintomáticamente. En cambio, los ítems de Falta de Confianza son codificados de manera inversa. Las propiedades psicométricas de las escalas fueron mencionadas más arriba.

\section{Resultados}

El coeficiente de Kaiser-Meyer-Olkin (=.870) y la prueba de esfericidad de Bartlett con valores 2639,70 (df: 435; sig = .000) sugirieron la posibilidad de llevar a cabo el Análisis Factorial, el cual se realizó a través del método de extracción de Ejes Principales. La regla de Kaiser-Guttman nos permitió identificar seis factores con autovalores mayores a 1, los que explicaron un $50 \%$ de la Varianza. Sin embargo, los resultados del Scree Plot (Cattell, 1966) y del Análisis Paralelo (Horn, 1965) indicaron una solución de cuatro factores que explicaron un $46 \%$ de la Varianza. Se realizó una rotación oblicua Promax eliminándose aquellos casos con correlación ítem-factor inferior a .35 (ver tabla 1).

Los resultados demostraron, por una parte, una clara mejora de los ítems 15 y 19 de interferencia y 6, 10 y 23 de preocupación, y por otra, que la reformulación del ítem 30 no tuvo ningún efecto por lo que no fue incluido de la versión final adaptada del TAI-G, al igual que el ítem 4, de saturación factorial inferior a .35.

Tabla 1 Matriz rotada de configuración

\begin{tabular}{llllll}
\hline & Item & $\mathbf{1}$ & $\mathbf{2}$ & $\mathbf{3}$ & $\mathbf{4}$ \\
\hline $\mathbf{1 -}$ & Tengo seguridad en mi capacidad & .762 & & \\
$\mathbf{8 -}$ & Tengo confianza en mi propio desempeño & .782 & \\
$\mathbf{1 3 -}$ & Se que puedo confiar en mi mismo & .832 & \\
$\mathbf{2 0 -}$ & Me siento conforme conmigo mismo & .695 & \\
$\mathbf{2 6 -}$ & Confío que lograre hacerlo todo & .633 & \\
$\mathbf{2 9 -}$ & Estoy convencido de que haré bien el examen & .771 & & \\
\hline
\end{tabular}


Continuación de la Tabla 1

\begin{tabular}{|c|c|c|c|c|c|}
\hline & Ítem & 1 & 2 & 3 & 4 \\
\hline 2- & Pienso en la importancia que tiene el examen para mí & & .566 & & \\
\hline 4- & Pienso sobre mi aptitud o talento & & & & \\
\hline 6- & Me preocupa saber si podré afrontarlo & & .358 & & \\
\hline 9. & Pienso en las consecuencias de fracasar & & .786 & & \\
\hline 10- & Me pregunto si mi rendimiento será lo suficientemente bueno & & .568 & & \\
\hline 14- & Pienso en lo mucho que me importa obtener un buen resultado & & .648 & & \\
\hline 17- & Me preocupo por el resultado de mi examen & & .736 & & \\
\hline 21- & Me preocupa cómo se verá mi calificación & & .624 & & \\
\hline 23- & Me preocupa que algo pueda salirme mal & & .566 & & \\
\hline 27- & Pienso en lo que pasará si me va mal & & .851 & & \\
\hline 3- & Tengo una sensación rara en mi estomago & & & .725 & \\
\hline $7-$ & Siento mi cuerpo tensionado & & & .789 & \\
\hline 12- & Me siento incómodo & & & .672 & \\
\hline 16- & Siento que mi corazón me late fuerte & & & .685 & \\
\hline 18- & Me siento ansioso & & & .526 & \\
\hline 22- & Tiemblo de nerviosismo & & & .636 & \\
\hline 25- & Tengo una sensación de angustia & & & .458 & \\
\hline 28- & Me siento nervioso & & & .849 & \\
\hline 5- & $\begin{array}{l}\text { De repente se me cruzan pensamientos por la cabeza que me } \\
\text { bloquean }\end{array}$ & & & & .352 \\
\hline 11- & Pienso en cualquier cosa y me distraigo & & & & .765 \\
\hline 15- & Fácilmente pierdo el hilo de mis pensamientos & & & & .694 \\
\hline 19- & $\begin{array}{l}\text { Otros problemas personales me interfieren y no me permiten } \\
\text { recordar las cosas. }\end{array}$ & & & & .401 \\
\hline 24- & $\begin{array}{l}\text { Me llama la atención algo de poca importancia que interrumpe } \\
\text { mi razonamiento }\end{array}$ & & & & .667 \\
\hline 30- & Me invade el pensamiento de que el examen está muy difícil. & & & & \\
\hline$\alpha$ & Escala Total: .90 & .86 & .87 & .88 & .74 \\
\hline
\end{tabular}

Nota: Se han suprimido los valores inferiores a .35 .

Por consiguiente, la versión final del GTAI-A (German Test Anxiety InventoryArgentinean Adaptation) quedó conformada por 28 ítems $(\alpha=.90)$ y cuatro escalas relacionadas con las dimensiones de la ansiedad frente a los exámenes conceptualizadas por 
Hodapp (1991): Falta de Confianza $(\alpha=.86)$, Preocupación $(\alpha=.87)$, Emocionalidad $(\alpha=.88)$ e Interferencia $(\alpha=.74)$. Las correlaciones entre las escalas son positivas y estadísticamente significativas, excepto entre Preocupación e Interferencia (ver tabla 2)

\section{Tabla 2.}

Matriz de intercorrelaciones

\begin{tabular}{lcccc}
\hline & 1 & 2 & 3 & 4 \\
\cline { 2 - 5 } 1- GTAI-A Total & - & & & \\
2- Interferencia & $.46^{* *}$ & - & & \\
3- Preocupación & $.76^{* *}$ & .08 & - & - \\
4- Falta De Confianza & $.60^{* *}$ & $.32^{* *}$ & $.19^{* *}$ & $.35^{* *}$ \\
5- Emocionalidad & $.83^{* *}$ & $.25^{* *}$ & $.51^{* *}$ &
\end{tabular}

Con la finalidad de obtener evidencias de validez de criterio del GTAI-A, se correlacionaron la puntuación total y de cada escala con el promedio de calificaciones autoinformado en la carrera de los participantes. La tabla 3 presenta los resultados de este último estudio.

\section{Tabla 3.}

Correlaciones con el promedio académico autoinformado

\begin{tabular}{lccccc}
\hline & Interferencia & Preocupación & Falta de Confianza & Emocionalidad & TAI-G \\
\hline $\begin{array}{l}\text { Promedio } \\
\text { autoinformado } \\
\text { de Calificaciones }\end{array}$ & $-.34 * *$ & .03 & $-.29 * *$ & -.06 & $-.18^{*}$ \\
\hline $\mathrm{p}>0.05 . * * \mathrm{p}>0.01$. & & & & &
\end{tabular}

Por último, se analizaron diferencias de género en relación a las puntuaciones totales y parciales del GTAI-A, utilizando la prueba $\mathrm{t}$ de diferencia de medias. Las mujeres presentaron puntajes significativamente más elevados en la escala total, Preocupación, Emocionalidad y Falta de Confianza. En Interferencia no se observaron diferencias significativas entre varones y mujeres. El tamaño del efecto ( $d$ de Cohen) observado en este análisis de diferencias de género fue, en general, medio (ver tabla 4). 


\section{Tabla 4.}

Diferencias de Género

\begin{tabular}{llllll}
\hline & \multicolumn{1}{l}{ Media Varones $(\mathrm{N}=33)$} & Media Mujeres $(\mathrm{N}=167)$ & $t$ & \multicolumn{1}{l}{$d$} \\
\cline { 2 - 6 } Interferencia & $8.60(3.29)$ & $8.58(2.65)$ & .048 & .01 & .962 \\
Preocupación & $23.51(6.27)$ & $26.83(5.65)$ & -3.02 & -.42 & .003 \\
Falta De Confianza & $12.48(3.72)$ & $14.59(3.70)$ & -2.99 & -.42 & .003 \\
Emocionalidad & $15.00(13.56)$ & $18.59(5.64)$ & -3.30 & -.47 & .001 \\
TAI-G Total & $59.69(13.56)$ & $68.60(12.13)$ & -3.77 & -.54 & .000 \\
\hline
\end{tabular}

\section{Discusión}

El proceso de adaptación a la población universitaria argentina del TAI-G permitió arribar a una versión que conserva la estructura original de cuatro factores bien definidos, con niveles adecuados de consistencia interna. Las correlaciones entre las escalas indicaron que todas las dimensiones se relacionan entre sí y la escala total, excepto la preocupación y la interferencia que no demostraron relaciones entre ellas. En este sentido es importante destacar que si bien, con el TAI-G es válido obtener una puntuación total de "ansiedad frente a los exámenes", existen relaciones específicas y diferenciales para cada una de las escalas con otras variables, tales como el afrontamiento o el rendimiento académico (Hodapp, 1996; Stöber, 2004).

Respecto de este último constructo se obtuvieron correlaciones negativas bajas o moderadas con las escalas Interferencia y Falta de Confianza, lo que concuerda sólo parcialmente con la literatura ya que la dimensión de la ansiedad que tradicionalmente se ha relacionado con el rendimiento es la Preocupación, y dicha escala en este caso, no demostró relaciones significativas con rendimiento. Una posible explicación de este hecho podría ser que los instrumentos utilizados en la mayoría de las investigaciones para medir la ansiedad frente a los exámenes, incluyen dentro del componente Preocupación ítems referidos a lo que en el TAI-G se denomina Interferencia y Falta de Confianza. Puede hipotetizarse, de acuerdo a lo que sugieren algunos autores, que la preocupación no ejerce necesariamente efectos negativos sobre el rendimiento, sino que podría tener un efecto motivador de conductas de afrontamiento centradas en el problema (Stöber, 2004) o estrategias auxiliares (Gutierrez Calvo, 1996), que permitan que el estudiante mantenga un buen rendimiento aún cuando 
experimente elevada ansiedad en las evaluaciones.

En cuanto a las evidencias de grupos contrastados, como la mayoría de las investigaciones lo indican (Hembree, 1988) las mujeres demostraron mayores niveles de ansiedad total, y de manera coincidente con los resultados obtenidos por Hodapp (1991) esta diferencia resultó consistente en casi todas las dimensiones del TAI-G, excepto Interferencia. Este hallazgo es relevante, ya que indica que las diferencias de género en ansiedad ante los exámenes se mantienen estables aún en contextos culturales diferentes, pero pueden observarse algunos matices, de acuerdo al instrumento elegido para su medición.

Por otra parte, la diferencia en las relaciones de la Interferencia y la Preocupación con el rendimiento académico y la ausencia de correlación entre estas dos dimensiones de la ansiedad frente a los exámenes plantea dos interrogantes: a) la relación entre la Preocupación y el rendimiendo está determinada por la presencia en las escalas de ítems referidos a la Interferencia?, y b), Interferencia es una dimensión de la ansiedad frente a los exámenes o una consecuencia de este último constructo?. Para responder a estas cuestiones, sería importante realizar investigaciones en las cuales se analicen los ítems que tradicionalmente se han incluido dentro de Preocupación así como identificar las variables asociadas al surgimiento de la Interferencia. A su vez, debería realizarse un análisis factorial confirmatorio en el cual se compare un modelo de cuatro factores que incluya Interferencia con otro de tres en donde se excluya esta dimensión.

Entre las limitaciones de este trabajo se destaca la necesidad de realizar análisis factorial confirmatorio para lo cual se requeriría una muestra de mayor tamaño a la utilizada en esta investigación (Boomsma \& Hoogland, 2001), como así también el desarrollo futuro de estudios que permitan evaluar la estabilidad del constructo. Otra limitación importante es la utilización de una muestra de participantes de una sola carrera (Psicología) de la Universidad Nacional de Córdoba. Estudios futuros deberían incluir estudiantes de diferentes disciplinas, universidades y regiones del país. Por otra parte, sería necesario elaborar baremos para interpretar comparativamente las puntuaciones obtenidas.

Desde un punto de vista práctico, es importante contar en nuestro medio con un instrumento multidimensional y confiable que podría ser utilizado en programas psicoeducativos o de intervención en la problemática de la ansiedad frente a los exámenes, mas aun teniendo en cuenta la prevalencia de esta problemática y los efectos que puede tener en la salud y el desempeño de los estudiantes en sus carreras. Desde una perspectiva teórica, 
el instrumento permitirá investigar modelos multivariados de rendimiento académico y bienestar psicológico de los estudiantes universitarios que incluyan el constructo de ansiedad frente a los exámenes junto a otros constructos relevantes, tales como estilos de afrontamiento, estrategias de aprendizaje y autoeficacia.

\section{Referencias}

Benson J. \& Bandalos D. (1992). Second-Order Confirmatory Factor Analysis of the Reactios To Test Scale with Cross Validation. Multivariate behavioral Research, 27, 3, 459-487.

Benson, J. \& Tippets, E. (1990). A confirmatory factor analysis of the Test Anxiety Inventory. En C.D. Spielberger \& R. Daz-Guererro (Eds.), Cross-cultural anxiety (Vol. 4,pp. 149-156). New York NY: Hemisphere/ Taylor-Francis

Benson, J. (1993). A Reconsideration of the Dimensionality of the Test Anxiety lnventory: A Case of Gender Diflerences. Paper presented at the 14th International Conference of the Stress and Anxiety Research Society, April 7, Cairo, Egypt.

Benson, J. \& El-Zahhar, N. (1994). Further refinement and validation of the Revised Test Anxiety Scale. Structural Equation Modeling, 1, 203 /221.

Boomsma A, Hoogland JJ. (2001) The robustness of LISREL modeling revisited. En: Cudeck R, Du Toit S, Sörbom D, editors. Structural equation modeling: present and future. A festschrift in honor of Karl Jöreskog. Chicago: Scientific Software International; . 139-68.

Buchwald, P. \& Schwarzer, C. (2004). Ein Messinstrument zur Prüfungsstressbewältigung: Das Multiaxiale Stressbewältigungsinventar für Prüfungssituationen (SBI-P) [An instrument for the assessment of coping with test stress: The German Strategic Approach to Coping Scale-Exam (GSACS-Exam)]. En: Buchwald, P., Schwarzer, C. and Hobfoll, S.E. (Eds.), Stress gemeinsam bewältigen: Ressourcenmanagement und multiaxiales Coping, pp. 74-88. Hogrefe, Göttingen, Germany.

Cattell, R. B. (1966). The scree test for the number of factors. Multivariate Behavioral Research, 1, $245-276$.

Cattell, R.B. \& Scheier, I.H. (1961). The Meaning and Measurement of Neuroticism and Anxiety . Ronald, New York.

Everson H., Millsap, R.E., \& Rodriguez, C .M. (1991). Isolating gender differences in test anxiety: A confirmatory factor analysis of the Test Anxiety Inventory. Educational and Psychological Measurement, 51, 243-251

Folin, O., Denis, W. \& Smillie, W.G. (1914). Some observationson “emotional glycosuria” in man. Journal of Biological Chemistry, 17, 519-520.

Gutiérrez-Calvo, M. \& Avero, P. (1995). Ansiedad, estrategias auxiliares y comprensión lectora: Déficit de procesamiento versus falta de confianza. Psicothema, 7, 569-578.

Heckhausen, H. (1982). Task-irrelevant cognitions during an exam: Incidence and effects. In: Krohne, H.W. and Laux, L. (Eds.), Achievement, Stress, and Anxiety, pp. 247-274. Hemisphere, Washington, DC.

Heredia D. \& Piemontesi S. (2008). Ansiedad frente a los examenes, estrategias de afrontameinto, autoeficacia 
para el aprendizaje autorregulado y rendiemitno academico. Tesis de licenciatura. Facultad de psicologia, Universidad Nacional de Cordoba. Inedito.

Hocevar, D. \& El-zahhar, N. (1985). Test anxiety in the USA and Egypt: A paradigm for investigating psychometric characteristics a cross cultures. In H.M. Van der Ploeg, R. Schwarzer, \& C.D. Spielberger (Eds.), Advances in Test Anxiety Research (vol. 4, pp). Lisse: Swets \& Zeitlnger.

Hodapp, V. \& Benson, J. (1997). The multidimensionality of test anxiety: A test of different models. Anxiety, Stress and Coping, 10, 219-244.

Hodapp, V. \& Henneberger, A. (1982). Test anxiety, study habits and academic performance. In H.M. van der Ploeg. R. Schwarzet, \& C. D. Spielberger (Eds.), Advances in Test Anxiety Research (Vol. 2, pp. 119127). Lisse: Swets \& Zeitlinger.

Hodapp, V. (1989). Anxiety, fear of failure, and achievement: Two pathanalyitical models. Anxiety Research. An International Journal, l, 301-312.

Hodapp, V. (1991). Das Prüfungsängstlichkeitsinventar TAI-G: Eine erweiterte und modifizierte Version mit vier Komponenten [The Test Anxiety Inventory TAI-G: An expanded and modified version with four components]. Zeitschrift für Pädagogische Psychologie , 5, 121-130.

Hodapp, V. (1995). The TAI-G: A multidimensional approach to the assessment of test anxiety. In: Schwarzer, C. and Zeidner, M. (Eds.), Stress, Anxiety, and Coping in Academic Settings, pp. 95 /130. Francke, Tübingen.

Hodapp, V. Laux, L., \& Spielberger C. D. (1982). Theorie und Messungder emotionalen und kognitiven Komponente der Prtifirngsangst (Theory and measurement of the emotional and cognitive component of test anxiety), Zeirchrift fur Diffrrentielle und Diagnostische Psychologie, 3, 169-184.

Hodapp, V., Glanzmann, P.G. \& Laux, L. (1995). Theory and measurement of test anxiety as a situationspecific trait. In: Spielberger, C.D. and Vagg, P.R. (Eds.), Test Anxiety. Theory, Assessment, and Treatment, pp. 47_/58. Taylor \& Francis, London.

Horn, J.L. (1965). A rationale and test for the number of factors in factor analysis. Psychometrika, 30, 179-185.

Keith, N., Hodapp, V., Schermelleh-Engel, K. \& Moosbrugger, H. (2003). Crossectional and longitudinal confirmatory factor models for the German Test Anxiety Inventory: A construct validation. Anxiety, Stress, and Coping, 16, 251-270.

Kerres, M. (1988). Prüfungsangst und-bewältigung. Eine Untersuchung zu selbstregulativen Aktivitaten im Prüfungsgeschehen. Frankfurt/ M: Lang

Liebert, R.M. \& Morris, L.W. (1967). Cognitive and emotional components of test anxiety: A distinction and some initial data. Psychological Reports , 20, 975-978.

Liepmann, D., Marggraf, C., Felfe, I., \&, Hosemann, A. (1992). Anxiety, action orientation, subjective state and situational aspects: A study of tank-lorry drivers. h K.A. Hagtvet \& T.B. Johnsen (Eds.), Advances in Test Aniety Research (vol. 7, pp. 130-141). Amsterdamllisse: Swets \& Zeitlinger

Musch, J. \& Broder, A. (1999). Uberprufung der psychometrischen Eigenschaften und der Validitat des multidimensionalen Prufungsangstlichkeitsinventars TAI-G [Investigating the psychometric properties and the validity of the multidimensional German Test Anxiety Inventory TAI-G]. Zeitschrift fur Padagogische Psychologie , 13, 100-105. 
Rost, D. H., \& Schermer, F. J. (1989). The various facets of test anxiety: A subcomponent model of test anxiety measurement. In R. Schwartzer, H. M. Van der Ploeg \& C. d. Spielberger (Eds.), Advances in test anxiety research, Vol. 6 (pp. 37-52 ). Lisse: Swets \& Zeitlinger, 37-52

Salamé , R. F. (1984). Test anxiety: Its determinants, manifestations, and consequences. In: van der Ploeg, H.M., Schwarzer, R. and Spielberger, C.D. (Eds.), Advances in Test Anxiety Research, pp. 83 /119. Swets \& Zeitlinger, Lisse.

Sarason, I.G. (1978) The Test Anxiety Scale: concept and research. In CD Spielberger \& I.G. Sarason (Eds) Stress and Anxiety, 5, 193-216. Washington DC Hemisphere

Sarason, S.B. \& Mandler, G. (1952). Some correlates of test anxiety. Journal of Consulting and Clinical Psychology, 47, 810-817.

Schwarzer, R. (Ed.). (1984). The self in anxiety, stress and depression. Amsterdam: North-Holland.

Schwarzer, R. \& Quast, H.H. (1985). Multidimensionalty of the anxiety experience: Evidence for additional components. In: van der Ploeg, H.M., Schwarzer, R. and Spielberger, C.D. (Eds.), Advances in Test Anxiety Research, pp. 3 /14. Swets \& Zeitlinger, Lisse.

Spielberger, C.D. (1966). Theory and research in anxiety. In: Spielberger, C.D. (Ed.), Anxiety and behavior, pp. 3 /20. Academic Press, New York.

Spielberger, C.D. (1980). Test anxiety inventory: Preliminary professional manual. Consulting Psychologists Press, Palo Alto, CA.

Stephan, E., Fischer, S., \& Stein, F. (1983). Self-related cognitions in test anxiety research: An empirical study and critical conclusions .In H.M. van der Ploeg, R . Schwarzer,\& C.D. Spielberger eds.), Advances in Test Anxiety Research( Vol. 2,pp. 45-66). Lisse: Swets\& Zeitlnger

Stöber, J. (2004), Dimensions of test anxiety: relations to ways of coping with pre-exam anxiety and uncertainty. Anxiety, Stress, and Coping, Vol. 17, No. 3, pp. 213-226

Ware, W., Galassi, J.P., Michie, K., \& Dew, H. (1990). The Test Anxiety Inventory: A confirmatory factor analysis. Aruiety Research. An International Journal, 3, 205-212

Wine, J. D. (1971). Test anxiety and direction of attention. Psychological Bulletin, 76, 92-104

Wine, J.D. (1982). Evaluation anxiety: A cognitive-attentional construct. In: Krohne, H.W. and Laux, L. (Eds.), Achievement, Stress, and Anxiety, pp. 207 /219. Hemisphere, Washington, DC.

Zeidner, M. \& Nevo, B. (1992). Test anxiety in examines in a college admission testing situation: krcidence, dimensionality, and cognitive correlates. In K.A. Hagvet \& T.B. Johnsen (Eds.), Advances in Test Anxiety Research (Vol. 7, pp. 288-303). Lisse: Swets\& Zetlinger.

Zimmer, J., Hocevar, D., Bachelor, P., \& Meinke, D.L. (1992). Art analysis of the Sarason( 1984) four-factor conceptualiz.aiono f test anxiety. kr K.A. Hagtvet \& T.B. Johnsen (Eds.), Advances in Test anxiety research (Vol. 7, pp. 103-113). Amsterdam Lisse: Swets\& Zetlinger 